\title{
Setup and Initial Results from the Magnetic Flux Surface Diagnostics at Wendelstein 7-X
}

\author{
M Otte ${ }^{1}$, D Aßmus ${ }^{1}$, C Biedermann ${ }^{1}$, S Bozhenkov ${ }^{1}$, T Bräuer ${ }^{1}$, A Dudek ${ }^{1}$, \\ J Geiger $^{1}$, G Kocsis ${ }^{2}$, S Lazerson ${ }^{3}$, T S Pedersen ${ }^{1}$, F Schauer ${ }^{1}$, T Szepesi ${ }^{2}$, \\ B. Standley ${ }^{1}$ and the W7-X Team \\ ${ }^{1}$ Max-Planck-Institut für Plasmaphysik, 17491, Greifswald, Germany \\ ${ }^{2}$ Wigner RCP, RMI, Konkoly Thege 29-33, H-1121, Budapest, Hungary \\ ${ }^{3}$ Princeton Plasma Physics Laboratory, Princeton, NJ 08540, USA
}

E-mail: matthias.otte@ipp.mpg.de

\begin{abstract}
Wendelstein 7-X is an optimized stellarator with superconducting magnetic field coils that just started plasma operation at Max-Planck-Institut für Plasmaphysik (IPP) Greifswald. Utilizing the electron beam technique the first vacuum flux surface measurements were performed during the commissioning of the magnet system. For the magnetic configurations investigated so far the existence of closed and nested flux surfaces has been validated. All features of the configuration designed for the initial plasma operation phase, including a predicted island chain, were confirmed. No evidence on significant magnetic field errors was found. Furthermore, the effect of the elastic deformation of the non-planar coils was confirmed by the measurements.
\end{abstract}

Keywords: vacuum magnetic flux surfaces, magnetic confinement, stellarator

PACS: 52.55.Dy, 52.55.Hc, 52.65.Cc, 52.70.Nc

\section{Introduction}

The existence of closed and nested magnetic flux surfaces is an essential prerequisite for magnetically confined plasma devices. In stellarator and torsatron magnetic configurations, the magnetic field confines charged particles even in the absence of plasma. Thus it is possible to determine the existence and quality of the magnetic flux surfaces for the vacuum case well before plasma operation. Furthermore, magnetic field errors arising due to imperfections in the manufacturing process, the alignment of the magnetic field coils during the assembly and the magnetic material in the vicinity of the magnet can be deduced and compensated e.g. by adjusting the magnetic configuration or installing additional error field coils can be initiated.

Wendelstein 7-X (W7-X) is a large fully optimized stellarator [1-3] with a mean major radius of $R=5.5 \mathrm{~m}$, a minor radius of $r=0.5 \mathrm{~m}$ and a plasma volume of about $30 \mathrm{~m}^{3}$ currently under 
commissioning at the Max-Planck-Institute for Plasma Physics in Greifswald, Germany [4,5]. The main magnet system consists of 50 non-planar superconducting coils of five different types (1-5) and 20 planar superconducting coils of two different types (A, B) in a five-fold toroidal periodicity. While the non-planar coils are mainly generating the required magnetic field strength and rotational transform $\mathrm{l}$, the planar coils allow to vary between the magnetic configurations. In addition 10 normalconducting sweeping coils integrated inside the plasma vacuum vessel and 5 normal-conducting trim coils outside the cryostat vacuum vessel, also arranged in five-fold toroidal periodicity, can be used to modify the magnetic edge topology and compensate field errors. Each module comprises two identical parts equipped with an island divertor unit to handle particle and thermal fluxes at the plasma edge. The edge topology of the magnetic flux surfaces with a rotational transform of $t_{a}=1 / 2 \pi=1$ results in a $n / m=5 / 5$ island chain of five independent islands, where $n$ and $m$ are the toroidal and poloidal mode numbers. By varying the current ratio in the non-planar and planar coils, the edge rotational transform can be varied between $0.7 \leq \mathfrak{t}_{\mathbf{a}} \leq 1.4$.

\section{Experimental Setup}

One of the optimization criteria for W7-X is the existence of closed and nested magnetic flux surfaces in a wide parameter range of the magnetic configuration. For the experimental proof of this optimization, a dedicated magnetic flux surface measurement (FSM) diagnostic has been designed, built, tested and installed [4-7]. The diagnostic principle is based on the spatial detection of a probing electron beam emitted along the magnetic field lines inside the confinement region. Electrons are generated by thermionic emission of a hot filament or crystal. A biasing voltage of typically a few tens of volts is applied between the emitter and the plasma vacuum vessel to accelerate the electrons. For the detection of the electrons and their spatial position, various methods were applied in the past. These can be divided into two groups. In the first one the spatial position of the toroidally and poloidally circulating electron beam is detected electrically by measuring directly the electric current by means of moveable (electrostatic) probes or wire frames [8-11]. The second group comprises indirect optical detection methods of the electron beam, either by interaction with a background gas in the plasma vacuum vessel or by the excitation of a fluorescent detector rod [12-18] intersecting the magnetic field lines and hence generating a Poincare plot. In addition, if the electron beam is exposed to a highly diluted background gas such as argon, nitrogen or hydrogen the whole three-dimensional structure of a field line is visible due to the inelastic collisional excitation. A potential application is the three dimensional in-situ metrology of magnetic field lines [19] and they can also be used as calibration source of other optical diagnostics.

Flux surface measurements are usually performed within a dedicated experimental campaign before the plasma operation whereby the diagnostic has to be first installed and later removed from the plasma vessel. However, there may be effects on the magnetic field system, such as permanent deformations of the magnetic-field coils, or a changing environment, due to the addition or removal of magnetic distortions. It is therefore advantageous to be able to perform flux surface measurements within an experimental campaign. It was therefore decided for W7-X to integrate the diagnostic permanently in the machine so that measurements can be performed anytime in-between plasma operation, without breaking vacuum. Furthermore, for the detection and estimation of possible magnetic error field which are expected in the lower $B_{\mathrm{nm}} / B_{0}=10^{-4}$ range it was requested to perform flux surface measurements up to a maximum magnetic field of $B_{0}=3.0 \mathrm{~T}$ and at two different toroidal positions [20]. Magnetic configurations with an edge rotational transform of $l_{a}=1$ are of special interest since they are prone to symmetry-breaking errors that are in resonance such as $n / m=1 / 1$ [21]. The latter point implies that one has either to exchange electron source and detection system or to design the diagnostic such that the operation mode can be selected conditionally. Aside from the before mentioned criteria, further constraints have to be met. Because of geometrical reasons measurements are favourable in an area where no plasma limiting components in the plasma vessel are installed. At W7-X this is the island divertor free region at the module interfaces. At this position, which is one of the two symmetry planes, the magnetic flux surfaces have a 'triangular' shaped cross 
section. The most suitable ports for the installation of such a diagnostic are the two so called AEV ports at every module interface. The ports are located on top and bottom of the plasma vessel and their port axes are directed to the same point in the centre of the plasma vessel at the so-called triangular plane at $\varphi=-36^{\circ}$ and $108^{\circ}$. However, they are not aligned in a vertical plane but are tilted by $24^{\circ}$ in toroidal and about $14^{\circ}$ in poloidal direction towards the centre of the machine.

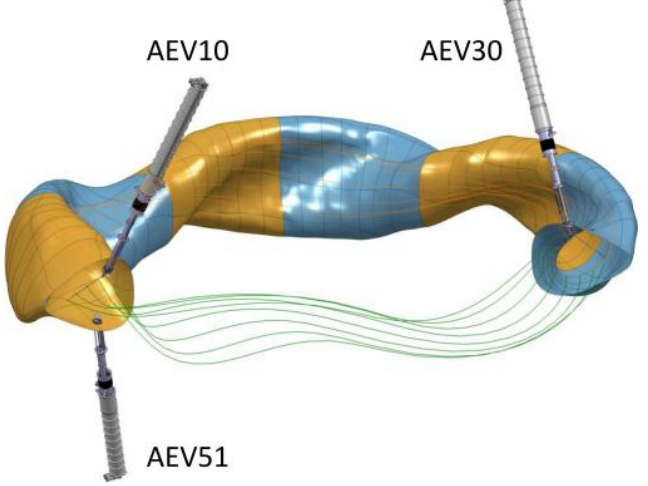

Figure 1. Cutaway drawing of the plasma vessel coloured in blue/brown with the three manipulators of the magnetic flux surface diagnostic at the ports AEV10, AEV30 and AEV51. In addition a single field line is shown in green.

For the integration of the flux surface diagnostic the ports AEV51 and AEV10 at the interface between module 5 and $1\left(\varphi=-36^{\circ}\right)$ and port AEV30 at the interface between module 2 and $3\left(\varphi=108^{\circ}\right)$ were chosen as shown in figure 1. By design the swivel plane for the rod detectors of the manipulators AEV51 and AEV10 is identical and can also be toroidally mapped onto the detection plane at AEV30.

\subsection{Electron emitter and detector system}

The electron emitter is the central element of the diagnostic. The cross-section of the electron gun which includes the emitter has to be as small as possible in order to minimize the unavoidable shadowing effect that comes from the electron beam hitting the back side of the e-gun after some number of toroidal turns. Especially near the magnetic axis, the field line under observation may hit the back side of the gun after just one or a few toroidal transits, thus preventing a clear verification of closed flux surfaces. During the development of the W7-X FSM diagnostic, various design variations and emitter materials (tungsten filament vs. $\mathrm{LaB}_{6}$ crystal) had been tested. $\mathrm{LaB}_{6}$ delivers higher electron emissivity under ultra-high vacuum conditions than tungsten, and, due to the low work function, it can be operated at a lower temperature. This reduces the amount of stray light which is emitted from the cathode into the plasma vessel. In the Wendelstein Experiment in Greifswald für die Ausbildung (WEGA) stellarator such a crystal survived an experimental campaign that lasted a few tens of hours in total and performed not only under optimal ultra high vacuum conditions but also with nitrogen, argon and hydrogen as background gas. A modified commercial $\mathrm{LaB}_{6}$ based emitter, originally foreseen for electron microscopy, was chosen. One of the integrated crystals has an emitting surface of about $0.4 \mathrm{~mm}$ in diameter at manipulator AEV30, a second one of $2.0 \mathrm{~mm}$ in diameter at manipulator AEV10. Both fit into the electron gun head made of a $12 \times 2 \mathrm{~mm}$ stainless steel tube. The emitters are electrically heated with a nominal power of about $10 \mathrm{~W}$. The required wires for heating and biasing are housed inside the hollow aluminium tube that fixes the gun. The emitted electrons are accelerated between the negatively biased emitter and the gun housing, which is on torus potential. The electron beam can exit the gun parallel to the magnetic field lines through an orifice that is $4 \mathrm{~mm}$ in diameter. Electrons moving in the opposite direction are absorbed by the outer cylindrical shell which only has an orifice in one direction. The exit cone for the electrons of about $\pm 30^{\circ}$ is large enough to cope with the varying orientation of the field lines depending on the local position and magnetic configuration. 


\subsection{Fluorescent detector}

Utilizing a fluorescent rod detector the electrons are imaged. The rod is made of a hollow $10 \times 2 \mathrm{~mm}$ aluminium tube with a total length of $883 \mathrm{~mm}$ including the electron gun and a fluorescent rod extension. The latter is situated beyond the electron gun and has an additional length of $94 \mathrm{~mm}$ and $3 \mathrm{~mm}$ in diameter. It is necessary for mapping the outer most flux surfaces and magnetic islands. The rod is fixed via a backlash-free joint to the manipulator and is covered with fluorescent $\mathrm{ZnO}: \mathrm{Zn}$ (commercial product name P15 or P24) powder [22]. The cathodoluminescent material has a low electron induced activation energy of a few $\mathrm{eV}$ only. Due to short decay time of the material in the $10 \mu$ s range no spatial smearing of the intersection points can be expected when moving the detector rod. The activated $\mathrm{ZnO}: \mathrm{Zn}$ emits in the visible range with a maximum at a wavelength of about $500 \mathrm{~nm}$ corresponding to the maximum quantum efficiency of present-day camera sensor technology.

\subsection{Manipulator}

For positioning of the electron gun and for swivelling the fluorescent rod detector two-axis manipulators are used. The rod can be driven in port-axial direction by $l=1450 \mathrm{~mm}$ and the joint allows a pivotal angle of $\alpha= \pm 70^{\circ}$ (compare to figure 2 (a)). During plasma operation the rod detector is completely retracted into the port behind a pneumatically driven shutter that protects against plasma and microwave stray radiation [20,23]. Each manipulator moves a fluorescent rod that carries in addition an electron gun as shown in figure 2 (b). Thus each unit can either be operated as electron source or detector.
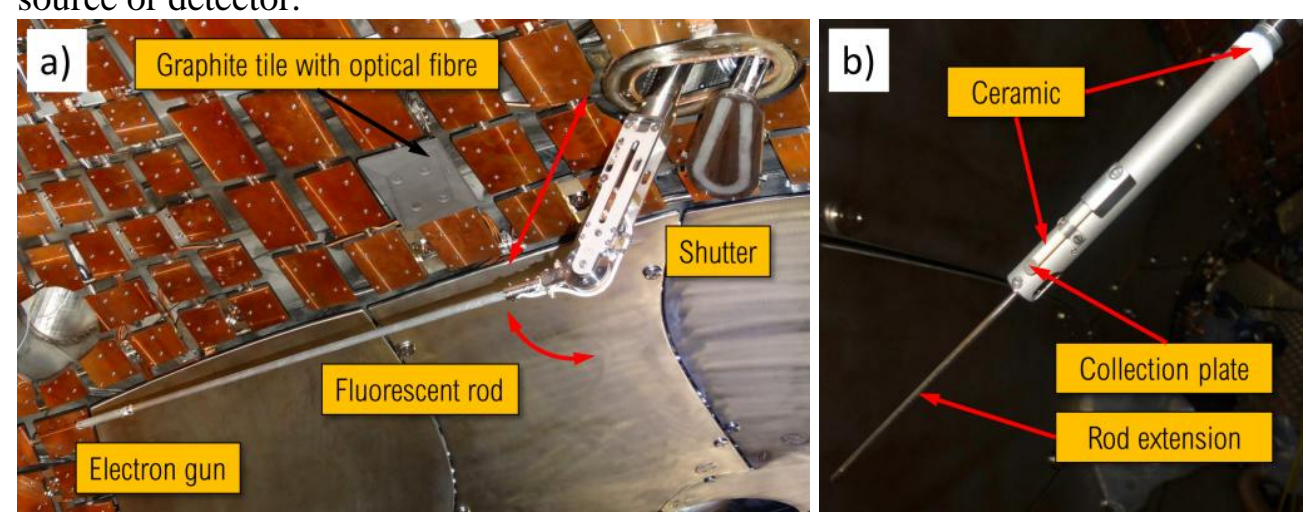

Figure 2. (a) Immersed manipulator components through opened shutter in W7-X plasma vessel. The fluorescent rod is rotated to the end position at $\alpha=-70^{\circ}$. The direction of motion of the rod is indicated by red coloured arrows. (b) Back side of the electron gun with rod extension, ceramic pieces and collection plate.

The manipulators are driven from outside the vacuum barrier by electric servo-motors, equipped with optical absolute encoders. The positioning of the electron gun is performed at a velocity of a few $\mathrm{mm} / \mathrm{s}$. The fluorescent rod is slowly rotated through the confinement region within $60 \mathrm{~s}$ to map the flux surface. The whole installation procedure was tested at a large vacuum tank at a port of nearly identical orientation as the on W7-X, and subsequent motion trials under vacuum conditions were performed. Three manipulators have been built and two of them have been integrated in ports AEV10 and AEV30. The third manipulator (for port AEV51) will be installed after the initial plasma operation phase (OP) 1.1. During the installation of the manipulators at W7-X the position of the electron gun and also the position and orientation of the plane defined by sweeping of the fluorescent rod were measured with respect to the global coordinate system utilizing an in-vessel laser tracker system. For this purpose the electron gun was replaced by a holder with three corner cubes [24]. The gathered information was included into the geometrical model description for the field line tracing calculations.

\subsection{Observation and reference system}


The observation system for the fluorescent light detection is composed of a PCO PixelFly $\mathrm{QE}^{\mathrm{TM}}$ grey scale cameras installed in the two tangential ports AEQ51 and AEQ21 looking at a distance of about $3.5 \mathrm{~m}$ nearly perpendicularly onto the detection plane of the manipulators. The charge coupled device (CCD) sensor has a resolution of $1392 \times 1024$ pixels and a maximum quantum efficiency of about $65 \%$ in the visible range. The cameras are installed on the atmosphere side behind a vacuum window and can thus be easily replaced by the fast complementary metal-oxide-semiconductor (CMOS) based event detection intelligent camera (EDICAM) cameras foreseen for the observation during plasma operation [25]. The camera in port AEQ51, which is located in the vicinity of the $140 \mathrm{GHz}$ electron cyclotron resonance (ECR) heating launcher system, is looking through a pinhole $5 \mathrm{~mm}$ in diameter that minimizes the microwave stray radiation onto the vacuum window and the camera system behind. In port AEQ21 the pinhole was omitted for OP 1.1 due to the expected reduced level of microwave stray radiation so that more light can be collected for this camera. Unless otherwise indicated, all further measurements presented were obtained at this position.

For scaling the images, and as a spatial calibration, an in-vessel reference system has been installed in both detection planes. Each one incorporates four copper-coated optical fibres of about $0.5 \mathrm{~mm}$ in diameter. The optical fibres are fixed on the first wall components and are inserted through pinholes in the graphite tiles, pointing towards the video camera. During the installation process, the exit position of the fibres was determined precisely by means of in-vessel metrology with an absolute precision of about $2 \mathrm{~mm}$ in the global W7-X coordinate system. The fibres are illuminated via an optical feedthrough flange by an external light emitting device (LED) light source.

Utilizing test images from the installed camera onto the reference system and the first wall components, the camera parameters such as the position, orientation, and the focal length have been determined after the pump out of the plasma vacuum vessel. With these parameter sets and additional images of the immersed manipulators their in-vessel position and orientation have been adapted with respect to the obtained metrology data during the installation process.

\subsection{Control and data acquisition system}

The power electronics for the servomotors, the Programmable Logic Controller (PLC), a control personal computer (PC) and the power supplies for the electron guns as well as the LED light source of the reference system are integrated within two electronic cabinets outside the torus hall. The whole diagnostic can be remotely controlled from the W7-X control room by a software package developed by the W7-X control and data acquisition group (CODAC). Control and visualization of the position and status of the manipulators, the power supplies for the electron gun and the light source for the reference system is realized by Siemens PLC hardware (Simatic S7-400) and software. All data generated by the FSM are stored in the W7-X archive database. Access to the data is granted via web based interfaces and various programming languages.

\subsection{Magnetic Configuration}

The magnetic system was operational up to an average field on axis of $B_{0}=2.5 \mathrm{~T}$ which is the resonant value for second harmonic ECR heating at $140 \mathrm{GHz}$. However, due to technical constraints, a reversal of the current in the planar field coils was not possible before OP 1.1. Thus, not all planned magnetic configurations are accessible yet. During the initial plasma experiments in OP 1.1, a poloidal inboard limiter is installed in each of the five 'bean' shaped symmetry planes protecting the first wall components from conductive heat loads. However, this also means that the edge islands in the 'standard' configurations for $n / m=4 / 5,5 / 5$ and $5 / 6$ are not accessible. The inertially cooled graphite tiles will be replaced after this operation phase by the test divertor unit (TDU). For the limiter operation, a new magnetic configuration was developed, with several $\mathrm{cm}$ radial range of good flux surfaces inside the last closed flux surface (LCFS) but without large magnetic edge islands and stochastic regions [7]. For a central magnetic field of $B_{0}=2.5 \mathrm{~T}$ a current of $I_{1-5}=12.8 \mathrm{kA}$ in all nonplanar coils type $1-5$ and a current of $I_{\mathrm{A}-\mathrm{B}}=5.0 \mathrm{kA}$ for the planar coils of type A and B has to be applied. The rotational transform with a central value of $\mathfrak{t}_{0}=0.79$ and $\mathfrak{t}_{a}=0.87$ at the plasma edge is 
lowered in contrast to the standard divertor configuration with an edge rotational transform of $\mathfrak{t}_{\mathrm{a}}=n / m=5 / 5=1$.

a)

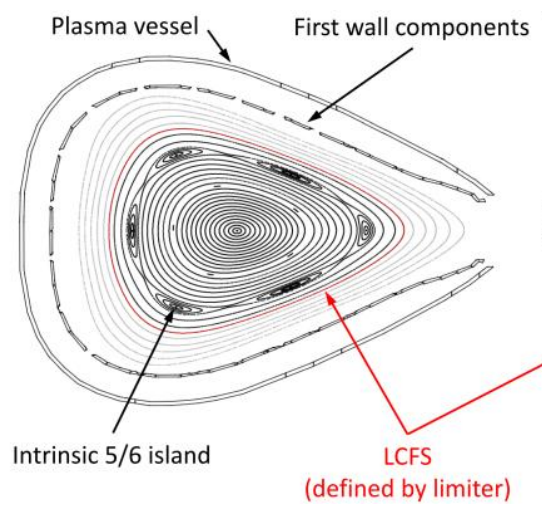

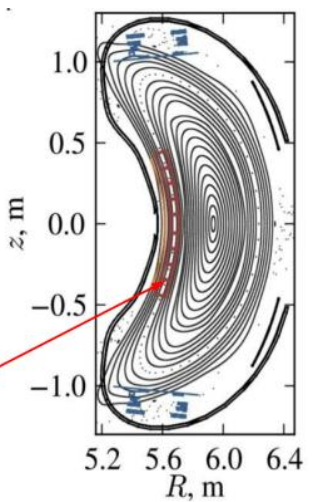

b)

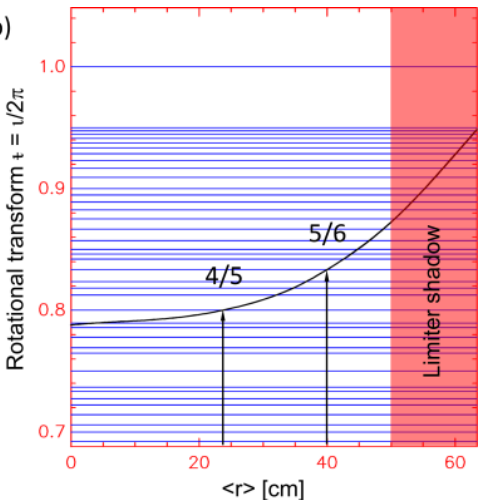

Figure 3. (a) Poincaré plot of the calculated OP 1.1 limiter configuration in the 'triangular' and 'bean' shaped symmetry plane with the limiter indicated. (b) Profile of the rotational transform for the OP 1.1 limiter configuration.

The reduced edge rotational transform makes the configuration relatively insensitive to low-order rational $n, m$ error fields. Two low-order rational resonances for $n / m=4 / 5$ and $n / m=5 / 6$ are located deeper inside the confinement region as well as shown in figure 3. Since W7-X is designed to have a finite $n=5$ component, but no $n=4$ Fourier component to its magnetic field, an $n / m=4 / 5$ island should not be present, but a prominent $5 / 6$ island chain is expected (see figure 3 (a)). The position, shape and size of the 5/6 island chain and the absence a 4/5 island chain can be used as an experimental indication of the quality and accuracy of this magnetic configuration, as we will show in the following.

\section{First Results from Flux Surface Measurements}

The primary goal for the first flux surface measurement campaign was the experimental proof of the existence of closed and nested flux surfaces and the validation of the magnetic configuration foreseen for the OP 1.1 plasma operation.

The first measurements were performed during the commissioning of the superconducting magnet system [5]. At that time, the plasma vessel was closed and had been under vacuum for a few weeks but not yet baked so that a base pressure on the order of $1 \times 10^{-6} \mathrm{mbar}$ was reached. The residual gas spectrum was at the time dominated by water vapour. During preparatory works of the electron gun in manipulator AEV10, the first field line became visible as shown in figure 4. The fluorescent rod detector at manipulator AEV30 was not inserted into the plasma vessel at that time so that purely the magnetic field lines are visualized.

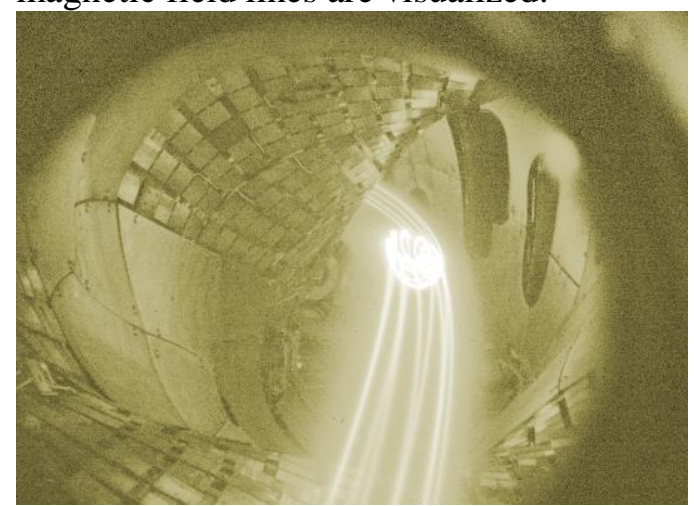

Figure 4. First light from a field line visualization (false colour) at W7-X. 
Seven toroidal transits of the electron beam, corresponding to a length of about $250 \mathrm{~m}$, are distinguishable with the camera in port AEQ21. The tangential observation of the field lines lets the anti-clockwise helical twist of the field lines, due to the rotational transform, become visible as well. The signal quality was enhanced by subtracting a background image and applying a nonlinear scale and a false colour table. Thus not only the field line but also the illuminated inner wall components and the ports are visible on the image. At an acceleration voltage of $U_{\mathrm{a}}=50 \mathrm{~V}$ an electron current of $0.25 \mathrm{~mA}$ inside the electron gun was measured. No additional gas source except the residual gas was present. The image was recorded with an exposure time of $65 \mathrm{~s}$.

By reducing the acceleration voltage to $U_{\mathrm{a}} \leq 20 \mathrm{~V}$ the excitation of the background gas could be reduced and the light emission due to interaction of the electron beam with the sweeping fluorescent rod starts to be dominating. With the given conditions the magnetic flux surfaces were recorded by changing the position of the electron gun at manipulator AEV10 with a step size of $2 \mathrm{~cm}$ and succeeding detection with the fluorescent rod detector at AEV30 again with an integration time of $65 \mathrm{~s}$ for the camera. An integrated flux surface of the configuration is obtained as shown in figure 5 for $B_{0}=0.4 \mathrm{~T}$ and $B_{0}=1.9 \mathrm{~T}$ by adding up the individual flux surfaces images from close to the magnetic axis to the LCFS. Good and nested flux surfaces could be detected without any stochastic regions or additional islands up to the LCFS is obtained for both cases. However, due to the presence of residual gas in the plasma vessel the number of distinguishable intersection points on a flux surface is limited to about 10 but the beam is smeared out over the whole surface. The finite size of the emission source leads due to the slightly varying rotational transform inside the beam cross section to an elliptical deformation of the initially circular emission cross section with increasing path length.
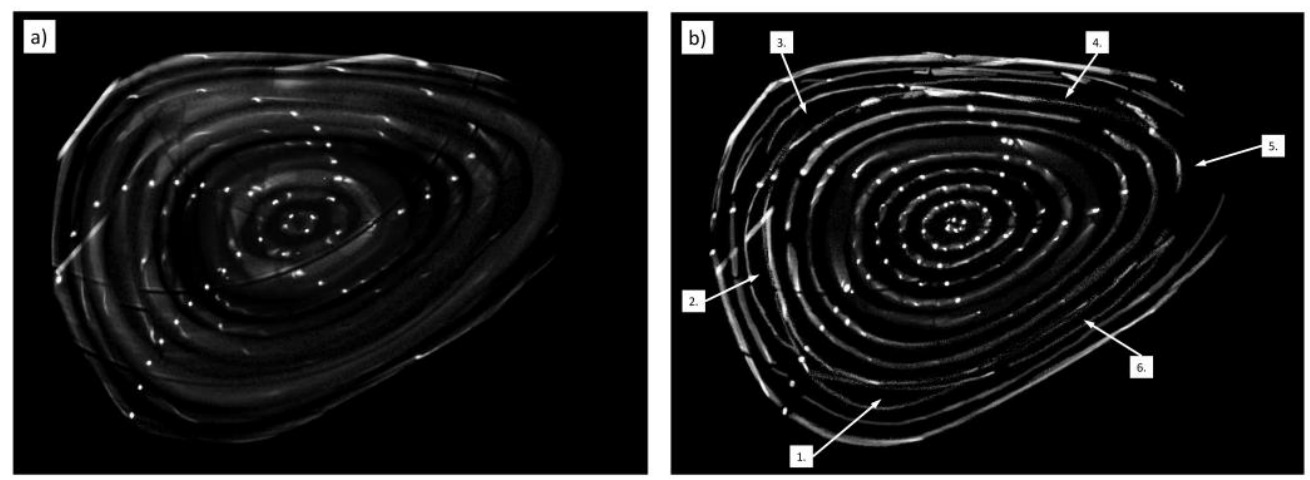

Figure 5. Magnetic flux surfaces for the OP 1.1 limiter configuration at $B_{0}=0.4 \mathrm{~T}$ (a) and $B_{0}=1.9 \mathrm{~T}$ (b) detected with the manipulator at the $\varphi=108^{\circ}$ position. The electron beam was created with the $\mathrm{LaB}_{6}$ crystal $2 \mathrm{~mm}$ in diameter installed in the manipulator at the $\varphi=-36^{\circ}$ position.

At low magnetic field strength of $B_{0}=0.4 \mathrm{~T}$, the magnetic surfaces appear thick and fuzzy as shown in figure 5(a). This is not an indication of stochastization of the field lines. Instead, we believe these are due to electrostatic effects. Charge accumulation of the emitted electrons can be excluded because the rods for positioning the electron gun and for the detection are grounded with respect to the plasma vessel. However, a short ceramic piece installed between the fluorescent rod and the electron gun as shown in figure 2(b) that allows an independent measurement of the electron current for the electron gun and the fluorescent rod may have been charged. In addition a small circular electrostatic plate for measuring the electron current when putting the electron gun emitter on the magnetic axis was mounted on the backside of the electron gun which was also electrically insulated by a ceramic tube from the rest of the electron gun. When the electron beam hits the ceramic pieces, the ceramic charges up negatively and thus shields itself from further electron flux. This negative charge electrostatically diffuses the electron beam via $E \times B$ onto the adjacent flux surfaces, in much the same way that nonneutral plasmas in a stellarator are transported radially from one flux surface to another flux surface by the presence of internal ceramic rods that charge up negatively [26, 27]. It has also been found that the 
presence of inserted ceramics prevent electron emitters from being accurately aligned to the same flux surface [28]. Space charge effects can occur both locally on the ceramic structures, and by the appearance of volumetric space charge, i.e. a pure electron plasma [26]. For future experiments, the ceramic pieces will be covered or removed with grounded conducting materials to avoid these effects. With the recorded position of the electron gun and the optimized parameters for the detection plane as input parameters numerical field line tracing simulations have been carried out.
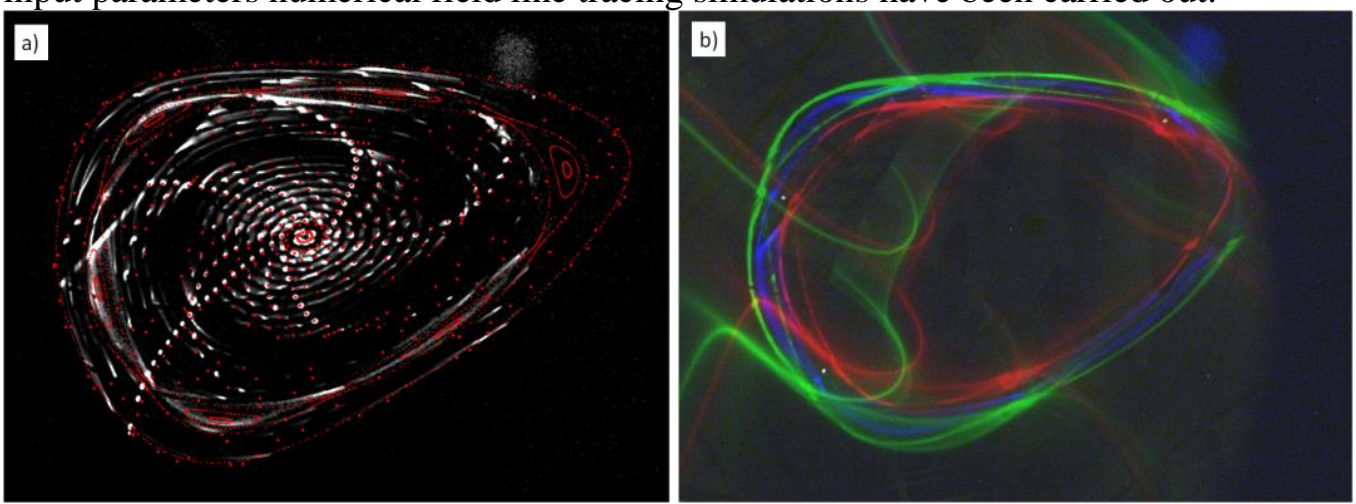

Figure 6. Poincaré plot (a) of the measured and calculated flux surfaces for the OP 1.1 limiter configuration at $B_{0}=1.9 \mathrm{~T}$. Superposition of the $5 / 6$ island chain (b) for a magnetic field strength $B_{0}$ of $0.4 \mathrm{~T}$ (red), $1.9 \mathrm{~T}$ (blue) and $2.5 \mathrm{~T}$ (green).

Figure 6 (a) presents an overlay of the measured magnetic flux surfaces at a field of $B_{0}=1.9 \mathrm{~T}$ (white structures) and the result from the magnetic field line tracing (red dots). The position and shape of the measured flux surfaces from the centre up to the LCFS are in good agreement with the simulations when using the geometry data of the as-built geometry. The aforementioned low order rational island chain at a rotational transform at $\mathfrak{t}=n / m=5 / 6$ can be identified thus qualitatively confirming the iota profile for this configuration. From a finite element modelling analysis (FEA) of the electromagnetic forces of the coils, small but important elastic deformations of the non-planar coils are expected for the nine standard configurations as the magnetic field strength is increased. With increasing magnetic field the non-planar coils have the tendency to deform into a more planar geometry associated with a decrease of the poloidal field component, thus lowering iota. In the experiments this effect became evident for the OP 1.1 limiter configuration when increasing the magnetic field $B_{0}$ from about $0.4 \mathrm{~T}$ to $2.5 \mathrm{~T}$ where a radial outward shift of the $n / m=5 / 6$ island chain by a few $\mathrm{cm}$ could be noticed as shown in figure $6(\mathrm{~b})$.

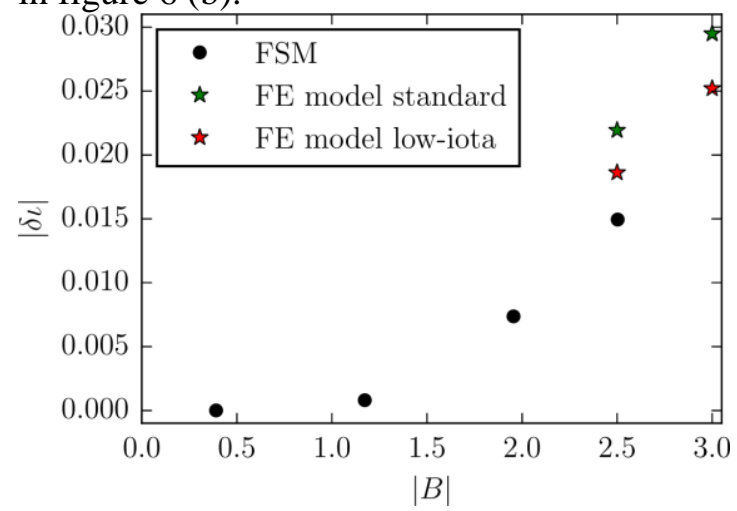

Figure 7. Variation of the rotational transform in dependence on the magnetic field strength and configuration. The measurements are indicated by the symbol ' $\bullet$ ', the results from FE analysis by ' $\star$ '.

Depending on the magnetic field strength and the magnetic configuration a decrease of the rotational transform of about $\mid \delta t+2 \%$ for a magnetic field of $B_{0}=2.5 \mathrm{~T}$ as shown in figure 6 was estimated for 
the magnetic 'standard' and 'low iota' configuration which have similar coil current ratios as the OP 1.1 limiter configuration.

However, for the new OP1.1 limiter configuration the FEA simulations still have to be performed. Instead the effect has been simulated by varying the current in the planar field coils while keeping the current in the non-planar coils fixed until a best fit in the position between the experimental and calculated intersection points was reached. The resulting decrease in rotational transform for the OP 1.1 limiter configuration was about $|\delta t|=1.5 \%$ as shown in figure 7 . For a more accurate comparison it is planned to repeat in a next step the field line tracing simulations with modified geometrical coil sets including the predicted deformation based on the FEA results.
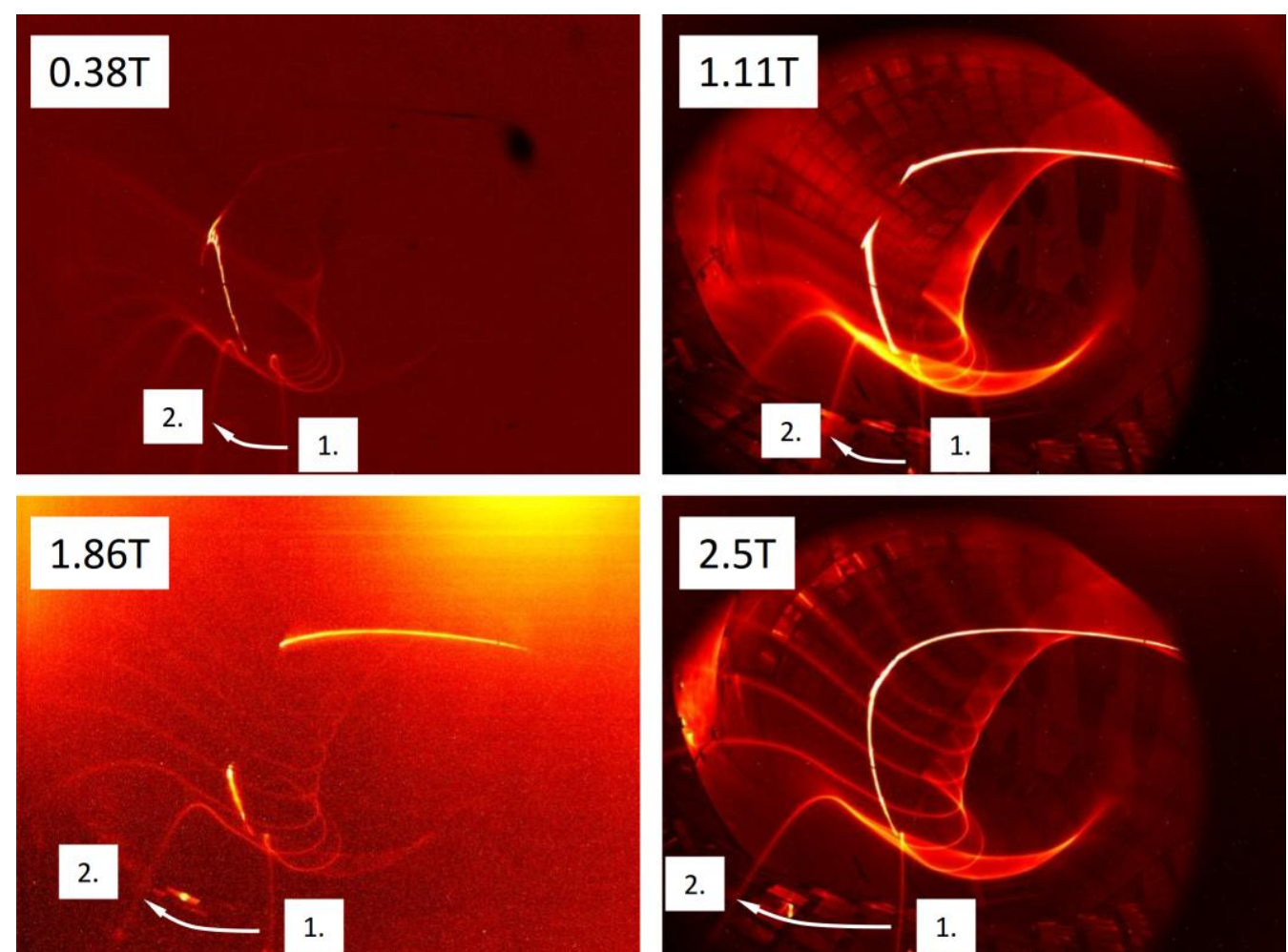

Figure 8. Field line visualization in residual gas for the 'standard' divertor configuration versus the magnetic field strength for a fixed electron emitter position. The poloidal angle between the first and second toroidal transits of the beam labelled with '1.' and '2.' is increasing with increasing field strength. The emitted electron current was about $0.5 \mathrm{~mA}$.

The effect of decreasing rotational transform in dependence on the magnetic field strength was also detected for other magnetic configurations. In figure 8, the visualized magnetic field lines for a fixed emitter position are shown for the 'standard' configuration, which has current in the non-planar coils only. It is clearly noticeable that the poloidal angular separation between the successive toroidal transits of the beam is increasing with increasing magnetic field strength. Since iota is slightly below 1 in this configuration, this indicates that the rotational transform is decreasing. Thus, the field line visualizations also confirm that the non-planar field coils exhibit a small elastic deformation in the presence of the magnetic field.

\section{Summary and Outlook}

For the vacuum flux surface measurements at W7-X a special diagnostic has been set up, based on the electron beam technique and an optical detection system. The positioning and movement of the electron beam and the detector are realized by remotely controlled manipulators. All data generated by the diagnostic are stored in the W7-X archive database and can be accessed via various interfaces. 
The initial results of the magnetic flux surface measurements confirm to the full extent the high expectations on the magnet system of W7-X. Good closed and nested flux surfaces were detected for various magnetic configurations and field strengths. The design features of the OP 1.1 limiter configuration dedicated for the initial plasma operation have been confirmed. The position, size and symmetry of the intrinsic $n / m=5 / 6$ island chain was detected as well. No indications of significant magnetic field errors have been found so far. The expected elastic deformation of the non-planar field coils and the associated decrease of the rotational transform could be quantitatively verified as well.

Further measurements are planned during OP 1.1 but also during the following operation phases when the third manipulator will also be operated. A detailed analysis is planned taking the slight geometry changes due to elastic deformations of the non-planar field coils into account for the OP1.1 configuration.

\section{Acknowledgments}

The authors gratefully acknowledge the work of the designers, engineers and technicians P. Lewioda, T. Bergmann, T. Broszat, D. Chauvin, K. Egorov, H. Greve, P. Grodzki, A. John, R. Laube, F. Kunkel, M. Köppen, P. Scholz, M. Schülke, H. Viebke, T. Xu as well as the manufacturing, assembly and CoDaC teams of Wendelstein 7-X.

This work has been carried out within the framework of the EUROfusion Consortium and has received funding from the Euratom research and training programme 2014-2018 under grant agreement No 633053. The views and opinions expressed herein do not necessarily reflect those of the European Commission.

\section{References}

[1] Beidler C D et al 1990 Fusion Technol. 17148

[2] Wobig H 1993 Plasma Phys. Control. Fusion 35 903-17

[3] Nührenberg J et al 1995 Trans Fusion Technol. 2771

[4] Bosch H S et al 2014 IEEE Trans. Plasma Sci. 42432

[5] Risse $\mathrm{K}$ et al 2016 Wendelstein 7-X - commissioning of the superconducting magnet system, IEEE Trans. Appl. Supercond. 264202004

[6] König R et al 2015 JINST 10 P10002

[7] Pedersen T S et al 2015 Nucl. Fusion 55126001

[8] Berezhetskii M S, Grebenshchikov S E, Popryadukhin A P, Shpigel' I S 1962 Sov. Phys.- Tech. Phys. 10, 1662 (Berezhetskii M S, Grebenshchikov S E, Popryadukhin A P, Shpigel' I S 1965 Zh. Tekh. Fiz. 35 2167)

[9] Sinclair R M, Hosea J C and Sheffield G V 1970 Rev. Sci. Instrum. 41 1552-9

[10] Lin H et al 1995 Rev. Sci. Instrum. 66 464-6

[11] Kumar S T A, Blackwell B D, and Harris J H 2007 Rev. Sci. Instrum. 78013501

[12] Colchin R J et al 1989 Rev. Sci. Instrum. 60 2680-9

[13] Lesnyakov G G et al 1992 Nucl. Fusion 32 2157-76

[14] Jaenicke R, Ascasibar E, Grigull P, Lakicevic I, Weller A and Zippe M 1993 Nucl. Fusion 33 687-704

[15] Ascasibar E, Qin J and Lopez-Fraguas A 1998 J. Plasma Fusion Res. 1 183-6

[16] Pedersen T S, Kremer J P, Lefrancois R G, Marksteiner Q, Sarasola X and Ahmad N. 2006 Phys. Plasmas 13012502

[17] Otte M et al 2008 AIP Conf. Proc. 993 3-10

[18] Morisaki T, Shoji M, Masuzaki S, Sakakibara S, Yamada H, Komori A and LHD Experiment Group 2010 Fusion Sci. Technol. 58 465-70

[19] Drewelow P, Bräuer T, Otte M, Wager F 2009 Rev. Sci. Instrum. 80123501

[20] Bosch H S et al 2013 Nucl. Fusion 53126001

[21] Andreeva T, Bräuer T, Endler M, Kißlinger J and v. Toussaint U 2009 Fusion Eng. Des. 84 408-12 
[22] Shionoya S et al 2006 Phosphor Handbook - second edition ed W M Yen, S Shionoya and H Yamamoto (Boca Raton: CRC Press) p 1080

[23] Eich T and Werner A 2008 Fusion Sci. Technol. 53 761-79

[24] Bräuer T, Mueller J, Otte M and John A 2015 On the Geometric Accuracy of Flux Surface Diagnostic installation, IEEE Trans. Plasma Sci. submitted

[25] Kocsis G et al 2015 Fusion Eng. Des. 96-97 808

[26] Kremer J P, Pedersen T S, Lefrancois, R G, Marksteiner Q 2006 Phys. Rev. Letters 97095003

[27] Berkery J W, Pedersen T S, Kremer J P, Marksteiner Q R, Lefrancois R G, Hahn M S, Brenner P W, et al 2007 Phys. Plasmas 14062503

[28] Hahn M S 2008 Pure Electron Plasma Equilibrium and Transport Jumps in the Columbia Non-Neutral Torus, Appendix B, PhD Thesis, Columbia University 